\title{
Evaluation de la fonction rénale chez les patients atteints de maladie cardio vasculaire (MCV) : comparaison entre la créatinine et la cystatine $C$
}

\author{
Massara CISSE-CAMARA ${ }^{1}$, Youzan Ferdinand DJOHAN ${ }^{1 *}$, \\ Amenan Germeine NIAMKE ${ }^{1}$, Luc DERE ${ }^{2}$, Gervais KOFFI ${ }^{1}$, Absalome Aké MONDE ${ }^{1}$, \\ Georges TIAHOU ${ }^{2}$ et Daniel SESS ${ }^{1}$
${ }^{1}$ Laboratoire de Biochimie médicale, UFR Sciences Médicales, Université Félix HOUPHOUET-BOIGNY de Cocody, Abidjan, Côte d'Ivoire.
${ }^{2}$ Laboratoire de Biochimie médicale, UFR Sciences Médicales, Université Alassane Ouattara de Bouaké, Côte d'Ivoire. \\ *Auteur correspondant ; E-mail : djohanferdinand@gmail.com ; Tél : 0022503227804
}

\section{RESUME}

L'évaluation de la fonction rénale est indispensable dans la prise en charge des néphropathies. L'objectif de l'étude était d'apprécier l'apport de la cystatine C dans l'évaluation de la fonction rénale. Il s'agissait d'une étude transversale réalisée chez 107 sujets des deux sexes, âgés de plus de 18 ans, répartis en deux groupes. Un groupe de 30 sujets apparemment sains et un groupe de 77 patients atteints de maladies cardiovasculaires reparti comme suit : 30 patients atteints d'hypertension artérielle, 28 atteints d'accident vasculaire cérébral et 19 atteints d'infarctus du myocarde. Les sérums, obtenus après traitement des prélèvements sanguins, ont servi au dosage des différents paramètres. Il ressort de l'étude que, pour les stades précoces de l'insuffisance rénale (IR), MDRD (Modification of diet in renal disease) sous-estime le débit de filtration glomérulaire (DFG) alors que la formule de Larsson le surestime; il existe une augmentation exponentielle significative plus nette pour la cystatine $C(p=0,0283)$ que pour la créatinine $(p=0,0534)$ en passant d'un stade de l'IR à un autre. La cystatinémie C est un meilleur marqueur du DFG que la créatininémie. Cependant, l'estimation du DFG par les formules basées sur la cystatine C ne présente pas d'avantage par rapport aux formules classiques basées sur la créatinine.

(C) 2016 International Formulae Group. All rights reserved.

Mots clés: Cystatine $\mathrm{C}$, créatinine, insuffisance rénale, débit de filtration glomérulaire, maladies cardiovasculaires.

\section{Evaluation of the renal function in patients with cardiovascular disease (CVD): comparison of creatinine and cystatin C}

\begin{abstract}
Assessment of renal function is indispensable in the treatment of kidney diseases. The objective of the study was to assess the contribution of cystatin $\mathrm{C}$ in the assessment of renal function. This was a crosssectional study in 107 subjects of both sexes, aged over 18 years, divided into two groups. A group of 30
\end{abstract}


apparently healthy subjects and a group of 77 patients with cardiovascular disease allocated as follows: 30 patients with high blood pressure, 28 with stroke and 19 with myocardial infarction. Serum obtained after treatment of blood samples, were used for the determination of the different parameters. According to the study: for the early stages of renal failure, MDRD underestimates GFR while the Larsson formula overestimates GFR; there is a sharper significant exponential increase for cystatin $C(p=0.0283)$ than creatinine $(p=0.0534)$ when passing from one stage to another. Serum cystatin $C$ is a better marker of GFR than creatinine. However, estimates of GFR by formulas based on cystatin C shows no advantage over conventional formulations based on creatinine.

(C) 2016 International Formulae Group. All rights reserved.

Keywords: cystatin C, creatinine, renal failure, glomerular filtration rate, cardiovascular disease.

\section{INTRODUCTION}

L'insuffisance rénale chronique (IRC) et les maladies cardiovasculaires (MCV) sont de véritable problèmes de santé publique (Coresh et al., 2007 ; Rosamond et al., 2007). Le risque de MCV est très élevé chez les patients insuffisants rénaux chroniques (Elsayed et al., 2007). En pratique courante, l'évaluation de la fonction rénale repose essentiellement sur la biologie clinique et plus particulièrement sur la mesure du débit de filtration glomérulaire (DFG). Le DFG est mesuré par la clairance de la créatinine qui nécessite le prélèvement des urines de 24 heures. Devant le caractère aléatoire de la récolte des urines de 24 heures, des formules d'estimation du DFG basées sur la concentration sérique de la créatinine ont été proposées. L'estimation du DFG chez l'adulte fait appelle à plusieurs formules parmi lesquelles la formule MDRD (Modification of diet in renal disease) simplifiée (Klahr et al., 1994) et la formule CKD-EPI (Chronic Kidney Disease Epidemiology collaboration) (Levey et al., 2009). La créatininémie étant influencée par l'âge, le sexe, l'alimentation et surtout la masse musculaire, ces formules ont certaines limites car se révèlent parfois insensibles à la détection de l'insuffisance rénale débutante chez les patients présentant des variations importantes de leur constitution corporelle (obésité, amyotrophie, etc.) (Poggio et al., 2005).

Ce constat d'insuffisance de la créatinine sérique motive la recherche de nouveaux marqueurs plasmatiques fiables. $\mathrm{La}$ cystatine $\mathrm{C}$, polypeptide basique, de bas poids moléculaire, de production constante, librement filtrée par le glomérule et non influencée par les facteurs rénaux, a été suggérée comme une alternative plus fiable (Dharnidharka et al., 2002 ; Roos et al., 2007). Cependant, des études récentes ont montré que la concentration plasmatique de la cystatine $\mathrm{C}$ est influencée par des facteurs tels que l'âge (Knight et al., 2004 ; Stevens et al., 2009), l'indice de masse corporelle (Knight et al., 2004 ; Macdonald et al., 2006), le sexe (Knight et al., 2004 ; Stevens et al., 2009), le tabagisme (Knight et al., 2004) et des concentrations élevées de la protéine Créactive (CRP) (Knight et al., 2004 ; Stevens et al., 2009).

Par analogie aux formules basées sur la créatininémie, plusieurs auteurs ont proposé des formules d'estimation du DFG basées sur la cystatinémie C. Parmi ces formules nous avons la formule de Larsson et al. (2004) et la formule de Rule et al. (2006).

L'objectif de cette étude était d'apprécier l'apport de la cystatine C dans l'évaluation de la fonction rénale chez une population atteinte de MCV et d'évaluer la performance des formules utilisant la créatinine et la cystatine $\mathrm{C}$.

\section{MATERIEL ET METHODES Patients}

Cette étude transversale a porté sur 107 sujets des deux sexes, âgés de plus de 18 ans, 
répartis en deux groupes. Un groupe de 30 sujets apparemment sains (22 hommes et 08 femmes) et un groupe de 77 patients atteints de maladies cardiovasculaires. Ce dernier groupe se composait comme suit : 30 patients (12 hommes et 18 femmes) atteints d'hypertension artérielle (HTA), 28 patients (16 hommes et 12 femmes) atteints d'accident vasculaire cérébral (AVC) et 19 patients (13 hommes et 06 femmes) atteints d'infarctus du myocarde (IDM). Le recrutement des sujets sains a porté sur une population tout venant en bonne santé apparente vivant à Abidjan. Les patients atteints de MCV ont été recrutés à l'Institut de Cardiologie d'Abidjan. Le protocole de l'étude a été approuvé par l'Unité de Formation et de Recherche des Sciences Médicales (UFRSM) de l'Université d'Abidjan - Cocody, et a été expliqué aux sujets avant qu'ils aient donné leur consentement bien éclairé.

\section{Prélèvements}

Les échantillons sanguins ont été prélevés chez des sujets à jeun depuis la veille au soir (12 heures), par ponction veineuse au pli du coude et recueillis dans des tubes vacutainer ${ }^{\circledR} \quad$ sans anticoagulant. Après centrifugation à 3000 tours par minute pendant cinq minutes, le sérum obtenu a été fractionné en aliquotes. Les aliquotes ont été conservées à $-20{ }^{\circ} \mathrm{C}$ jusqu'au moment des analyses.

\section{Dosage}

Le cholestérol total, les triglycérides et la créatinine ont été dosés par les méthodes enzymatiques usuelles. Le cholestérol HDL a été dosé après précipitation des autres lipoprotéines par le phosphotungstate. La valeur du cholestérol des LDL a été obtenue par calcul selon la formule de Friedewald (1972). La CRP a été dosée par immunonéphélémétrie et la cystatine $\mathrm{C}$ par immunoturbidimétrie. Toutes ces analyses ont été réalisées sur l'automate multiparamétrique Hitachi 917® (Roche Diagnostics, Mannheim, Allemagne).

Les valeurs de créatinine et de cystatine $\mathrm{C}$ ont servi à déterminer le DFG en utilisant différentes formules (Tableau 1). Pour la classification des sujets selon le stade de l'insuffisance rénale (IR), les lignes directrices de la NKF (National Kidney Foundation) ont été utilisées (Levey et al., 2003) (Tableau 2).

\section{Analyse statistique}

Le traitement des données recueillies a été réalisé à l'aide du logiciel Epi-info version 6.0. Les résultats ont été exprimés en moyenne \pm écart type. La comparaison des moyennes a été réalisée par le test paramétrique de Student. Le test a été considéré comme significatif pour une valeur de $\mathrm{p}<0,05$.

\section{RESULLTATS}

Les résultats de cette étude sont consignés dans les Tableaux 3,4 et 5 . On observe une augmentation significative de la cystatine $\mathrm{C}$ et de la CRP chez les patients atteints d'AVC et d'HTA $(p<0,05)$ (Tableau 3). La stratification des stades de l'IR montre que MDRD sous-estime le DFG pour les stades précoces alors que les stades terminaux sont plus sous-estimés par les approches basées sur la cystatine C. La formule de Larsson surestime le DFG normal ou élevé (Tableau 4). La comparaison des variations de la cystatine $\mathrm{C}$ et de la créatinine selon les stades de l'IR, montre une augmentation exponentielle significative de la cystatine $\mathrm{C}(\mathrm{r}$ $=0,977)$ et de la créatinine $(\mathrm{r}=0,979)$. Cette augmentation est plus nette, en passant d'un stade à l'autre, pour la cystatine $\mathrm{C}$ que pour la créatinine (Tableau 5). 
Tableau 1 : Formules adoptées pour le calcul du DFG.

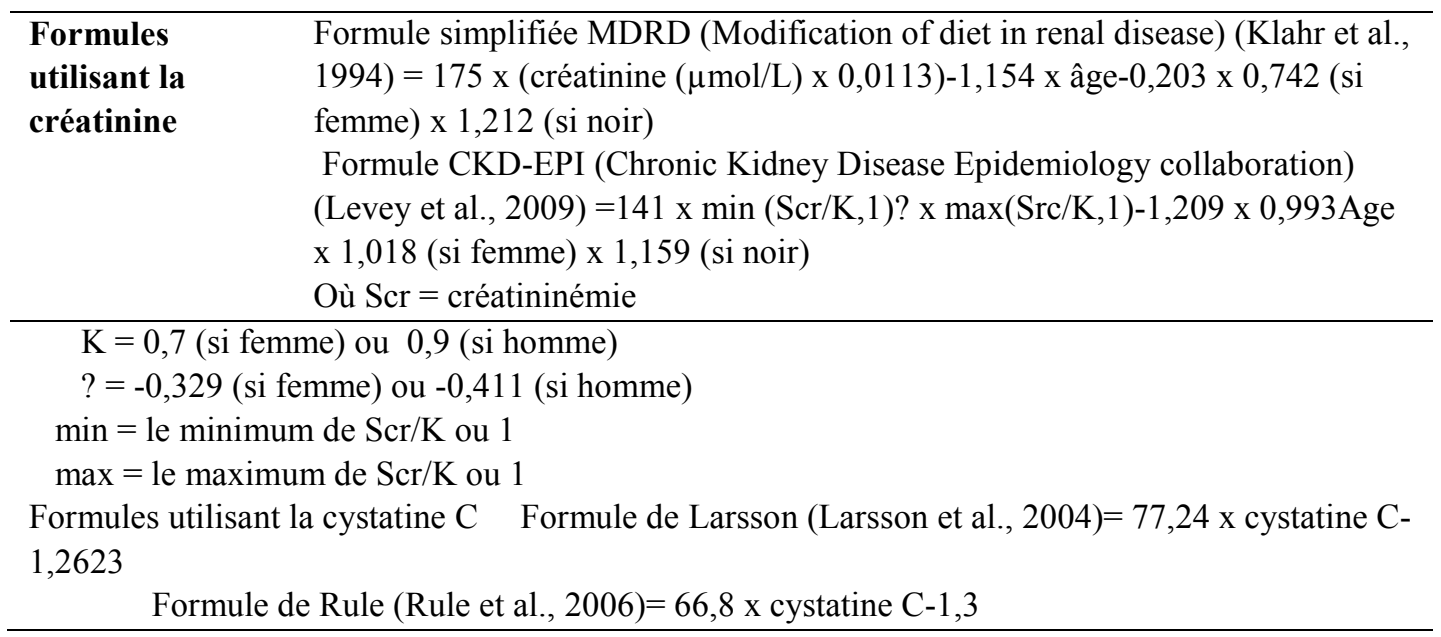

Tableau 2 : Classification de l'insuffisance rénale selon la NKF (Levey et al., 2003).

\begin{tabular}{lll}
\hline Stades & Définitions & $\begin{array}{l}\text { DFG } \\
(\mathbf{m L} / \mathbf{m i n} / \mathbf{1 , 7 3 m} \mathbf{m})\end{array}$ \\
\hline 1 & Pas d'insuffisance rénale* & 90 \\
2 & Insuffisance rénale légère & $60-89$ \\
3 & Insuffisance rénale modérée & $30-59$ \\
4 & Insuffisance rénale sévère & $15-29$ \\
5 & Insuffisance rénale terminale & $<15$ \\
\hline DFG = Débit de filtration glomérulaire ; *Atteinte rénale se manifestant par des anomalies histologiques et/ou \\
biologiques et/ou morphologiques.
\end{tabular}

Tableau 3 : Paramètres biochimiques analysés dans la population d'étude.

\begin{tabular}{|c|c|c|c|c|}
\hline Paramètres & Témoins $(\mathrm{n}=30)$ & $\overline{A V C}$ & & \\
\hline$(n=28)$ & HTA & & & \\
\hline$(n=30)$ & IDM & & & \\
\hline$(n=19)$ & & & & \\
\hline $\begin{array}{l}\text { Cholestérol } \\
(\mathrm{mmol} / \mathrm{L})\end{array}$ & $5,16 \pm 0,24$ & $4,32 \pm 0,50^{*}$ & $5,21 \pm 0,31$ & $2,86 \pm 0,05^{*}$ \\
\hline $\begin{array}{l}\text { Triglycérides } \\
(\mathrm{mmol} / \mathrm{L})\end{array}$ & $1,01 \pm 0,49$ & $1,24 \pm 0,06$ & $1,09 \pm 0,78$ & $1,02 \pm 0,06$ \\
\hline $\mathrm{HDLc}(\mathrm{mmol} / \mathrm{L})$ & $1,12 \pm 0,52$ & $0,72 \pm 0,42 *$ & $1,18 \pm 0,76$ & $0,69 \pm 0,04^{*}$ \\
\hline LDLc (mmol/L) & $3,58 \pm 0,06$ & $3,04 \pm 0,11$ & $3,53 \pm 0,09$ & $1,71 \pm 0,03^{*}$ \\
\hline CRP (mg/L) & $1,33 \pm 0,49$ & $65,74 \pm 63,64^{*}$ & $11,59 \pm 13,58^{*}$ & $6,41 \pm 0,64$ \\
\hline $\begin{array}{l}\text { Cystatine C } \\
(\mathrm{mg} / \mathrm{L})\end{array}$ & $0,81 \pm 0,01$ & $1,08 \pm 0,37^{*}$ & $1,10 \pm 0,36^{*}$ & $0,90 \pm 0,13$ \\
\hline $\begin{array}{l}\text { Créatinine } \\
(\mu \mathrm{mol} / \mathrm{L})\end{array}$ & $94,73 \pm 11,31$ & $118,29 \pm 91,92 *$ & $110,80 \pm 9,90$ & $110,63 \pm 36,77$ \\
\hline
\end{tabular}


Tableau 4 : Stratification des stades de l'insuffisance rénale selon les différentes approches.

\begin{tabular}{|c|c|c|c|c|}
\hline $\begin{array}{l}\text { Approches } \\
\text { basées sur la } \\
\text { créatinine }\end{array}$ & & $\begin{array}{l}\text { Approches } \\
\text { basées sur la } \\
\text { cystatine } C\end{array}$ & & \\
\hline Stades de l'IR & $\begin{array}{l}\text { MDRD simplifié } \\
\text { CKD-EPI }\end{array}$ & $\begin{array}{l}\text { Formule } \\
\text { de Rule }\end{array}$ & $\begin{array}{l}\text { Formule de } \\
\text { Larsson }\end{array}$ & \\
\hline Stade 1 & $34,6 \%$ & $42,1 \%$ & $36,4 \%$ & $51,4 \%$ \\
\hline Stade 2 & $42 \%$ & $36,5 \%$ & $39,3 \%$ & $30 \%$ \\
\hline Stade 3 & $18,7 \%$ & $16,8 \%$ & $21,5 \%$ & $16,8 \%$ \\
\hline Stade 4 & $4,7 \%$ & $3,7 \%$ & $1,9 \%$ & $0,9 \%$ \\
\hline Stade & $0 \%$ & $0,9 \%$ & $0,9 \%$ & $0,9 \%$ \\
\hline
\end{tabular}

Tableau 5 : Variation de la créatinine et de la cystatine C selon les stades de l'insuffisance Rénale.

\begin{tabular}{lclllll}
\hline & $\begin{array}{l}\text { Stade } \\
\mathbf{1}(\mathbf{n}=\mathbf{4 5})\end{array}$ & $\begin{array}{l}\text { Stade 2 } \\
(\mathbf{n = 3 9 )}\end{array}$ & $\begin{array}{l}\text { Stade 3 } \\
(\mathbf{n = 1 8})\end{array}$ & $\begin{array}{l}\text { Stade 4 } \\
(\mathbf{n = 4 )}\end{array}$ & $\mathbf{r}$ & $\mathbf{p}$ \\
\hline $\begin{array}{l}\text { Créatinine } \\
(\mu \mathrm{mol} / \mathrm{L})\end{array}$ & $77 \pm 6$ & $105 \pm 10$ & $127 \pm 14$ & $291 \pm 124$ & 0,979 & $<0,002$ \\
$\begin{array}{l}\text { Cystatine C } \\
(\mathrm{mg} / \mathrm{L})\end{array}$ & $0,83 \pm 0,05$ & $0,91 \pm 0,16$ & $1,06 \pm 0,11$ & $2,15 \pm 0,79$ & 0,977 & $<0,001$ \\
$\begin{array}{l}\text { Comparaison entre stades } \\
\text { Créatinine }\end{array}$ & Cystatine C & & & & \\
\multicolumn{7}{c}{} \\
Stade 1 & vs stade 2 & 0,0421 & 0,0149 & & & \\
Stade 1 & vs stade 3 & 0,0254 & 0,0039 & & & \\
Stade 1 & vs stade 4 & 0,0138 & 0,0014 & & \\
Stade 2 & vs stade 3 & 0,0484 & 0,0283 & & \\
Stade 2 & vs stade 4 & 0,0294 & 0,0023 & & \\
Stade 3 & vs stade 4 & 0,0534 & 0,0081 & & \\
\hline
\end{tabular}

\section{DISCUSSION}

L'augmentation de la cystatine $\mathrm{C}$ et de la CRP étaient statistiquement significative chez les patients atteints d'AVC et d'HTA (p $<0,05$ ) (Tableau 3). La cystatine $C$ et la CRP sont de plus en plus utilisées comme des facteurs de risque cardiovasculaires. L'utilisation de la CRP comme facteur de risque cardiovasculaire est due à son implication dans le processus athéromateux (Calabro et al., 2003).

Pour des valeurs du DFG 90 $\mathrm{mL} / \mathrm{min} / 1,73 \mathrm{~m} 2 \mathrm{MDRD}$ et la formule de Rule sous-estiment le DFG alors que CKDEpi et la formule de Larsson le surestiment. Alors que pour des valeurs du DFG situées entre 60 et $89 \mathrm{~mL} / \mathrm{min} / 1,73 \mathrm{~m} 2$, c'est le contraire qui est observé; MDRD et la formule de Rule surestiment le DFG alors que CKD-Epi et la formule de Larsson le sousestiment. Pour des valeurs du DFG $<60$ $\mathrm{mL} / \mathrm{min} / 1,73 \mathrm{~m} 2$, seule la formule de Larsson a sous-estimé le DFG (Tableau 4). Cette sousestimation du DFG par MDRD pour des valeurs normales ou élevées, a été mise en évidence par de nombreux auteurs ( $\mathrm{Li}$ et al., 2010; Ben Dhia et al., 2012). Earley et al. (2012) ont montré que les formules MDRD et CKD-EPI ont une fiabilité équivalente. Cependant, la formule CKD-EPI serait un meilleur indice quant au pronostic (Stevens et al., 2011; Matsushita et al., 2012).

Il ressort que les formules basées sur la cystatine $\mathrm{C}$ présentent en leur sein des 
performances différentes. Ces formules ont souvent été élaborées à partir d'échantillons trop petits et/ou de population trop spécifique (Séronie-vivien et al., 2008). Larsson et al. (2004) ont établi leur formule basée sur la cystatine $\mathrm{C}$ à partir d'un échantillon de 100 sujets caucasiens (40 femmes et 60 hommes) âgés de 4 à 92 ans. Rule et al. (2006) ont travaillé sur 204 sujets caucasiens atteints en général de maladies rénales chroniques $(92$ femmes et 112 hommes).

La comparaison des variations de la cystatine $\mathrm{C}$ et de la créatinine selon les stades de l'IR, montre une augmentation exponentielle significative de la cystatine $\mathrm{C}(\mathrm{r}$ $=0,977)$ et de la créatinine $(r=0,979)$. Cette augmentation est plus nette, en passant d'un stade à l'autre, pour la cystatine $\mathrm{C}$ que pour la créatinine. La cystatine $\mathrm{C}$ augmente très significativement lors du passage d'un stade à un autre $(\mathrm{p}=0,0283)$. La créatinine augmente aussi significativement lors du passage d'un stade à un autre $(\mathrm{p}=0,0484)$ sauf pour le passage du stade 3 au stade $4(\mathrm{p}=0,0534)$ (Tableau 5). Ben Dhia et al. (2012) ont retrouvé une augmentation très significative de la cystatine $\mathrm{C}$ lors du passage d'un stade à un autre avec $\mathrm{p}<0,001$, plus que la créatinine avec $\mathrm{p}<0,01$. Ils ont également retrouvé une augmentation non significative de la créatinine $(\mathrm{p}=0,05)$ en passant du stade 3 au stade 4. Shimizu et al. (2003) ont rapporté la présence d'une association significative entre la cystatine $\mathrm{C}$ et les stades de l'insuffisance rénale $(\mathrm{p}<0,0005)$ contrairement à la créatinine dont l'augmentation n'était significative que pour les passages des stades 1 et 2 au stade 4. Yang et al. (2007) ont constaté que la cystatine $\mathrm{C}$ augmentait significativement lors du passage du stade 1 vers le stade 2 et du stade 1 vers le stade 3, alors que la créatinine sérique augmentait significativement uniquement lors du passage du stade 2 vers le stade 3 .

De nombreux auteurs ont mise en évidence la meilleure sensibilité de la cystatinémie $\mathrm{C}$ par rapport à la créatininémie pour la détection précoce de l'insuffisance rénale chronique (Dharnidharka et al., 2002; Roos et al., 2007). Pour ce qui est des formules basées sur la cystatinémie $\mathrm{C}$, leur avantage apparaît, au jour d'aujourd'hui, relativement faible par rapport aux formules MDRD et CKD-EPI basées sur la créatininémie, l'âge, le sexe et la race, en ce qui concerne la population générale (Beauvieux et al., 2007; Herget-Rosenthal et al., 2007) et leur précision semble aussi limitée (Beauvieux et al., 2007 ; Zahran et al., 2007). Devant cet état de fait, certains auteurs recommandent des formules combinant la créatinine et la cystatine C (Ma et al., 2007; Stevens et al., 2008).

Il ressort de cette étude que la cystatinémie $\mathrm{C}$ est un bon marqueur du DFG avec une sensibilité supérieure à celle de la créatininémie. Tout comme la créatinine sérique, la cystatine $\mathrm{C}$ ne donne pas, en ellemême, une bonne estimation du DFG. De nombreuses formules d'estimation du DFG basées sur la cystatinémie $\mathrm{C}$ ont été proposées. Cependant, des études de validation à grande échelle portant sur des populations plus ciblées ainsi que des études longitudinales seront encore nécessaires pour affirmer l'intérêt de ces formules dans l'estimation du DFG.

\section{Conclusion}

La cystatinémie $\mathrm{C}$ est un bon marqueur du DFG avec une sensibilité supérieure à celle de la créatininémie. Cependant, l'estimation du DFG par les formules basées sur la cystatine $\mathrm{C}$ ne présente pas d'avantage par rapport aux formules classiques basées sur la créatinine. Au vu de la différence de prix (le coût du dosage de la cystatine $\mathrm{C}$ reste dix fois plus élevé que celui de la créatinine), les formules d'estimation du DFG basées sur la créatinine restent toujours d'actualité.

\section{CONFLIT D'INTERET}

Il n'existe aucun conflit d'intérêt entre les auteurs de ce travail. 


\section{CONTRIBUTION DES AUTEURS}

YFD: Conception et mise au point de l'étude, analyse et interprétation des données Ecriture de l'article, approbation finale de l'article; MCC: Conception et mise au point de l'étude, analyse et interprétation des données, approbation finale de l'article; AGNA: Conception et mise au point de l'étude, analyse et interprétation des données, approbation finale de l'article; GK: Analyse et interprétation des données, approbation finale de l'article; LD: Analyse et interprétation des données, approbation finale de l'article; AAM: Conception et mise au point de l'étude, approbation finale de l'article; GT: Conception et mise au point de l'étude, approbation finale de l'article; DS: Conception et mise au point de l'étude, approbation finale de l'article.

\section{REMERCIEMENTS}

Nous tenons à remercier le personnel de l'institut de cardiologie d'Abidjan ainsi que les techniciens du Laboratoire de Biochimie du CHU de Cocody pour leur précieuse collaboration.

\section{REFERENCES}

Beauvieux MC, Le Moigne F, Lasseur C, Raffaitin C, Perlemoine C, Barthe N, Chauveau P, Combe C, Gin H, Rigalleau V. 2007. New predictive equations improve monitoring of kidney function in patients with diabetes. Diabetes Care, $\mathbf{3 0}$ : 1988-1994.

Ben Dhia R, Hellara I, Harzallah O, Neffati F, Khochtali I, Mahjoub S, Najjar MF. 2012. Évaluation de la fonction rénale chez le diabétique type 2: calcul des clairances ou cystatine C? Ann. Biol. Clin., 70(3): 287294.

Calabro P, Willerson JT, Yeh ET. 2003. Inflammatory cytokines stimulated $\mathrm{C}$ reactive protein production by human coronary artery smooth muscle cells. Circulation, 108(16): 1930-1932.
Coresh J, Selvin E, Stevens LA, Manzi J, Kusek JW, Eggers P, Van Lente F, Levey AS. 2007. Prevalence of chronic kidney disease in the United States. JAMA, 298: 2038-2047.

Dharnidharka VR, Kwon C, Stevens G. 2002. Serum cystatin $\mathrm{C}$ is superior to serum creatinine as a marker of kidney function: a meta-analysis. Am. J. Kidney Dis., 40: 221-226.

Earley A, Miskulin D, Lamb EJ, Levey AS, Uhlig K. 2012. Estimating equations for glomerular filtration rate in the era of creatinine standardization : a systematic review. Ann. Intern. Med., 156: 785-795.

Elsayed EF, Tighiouart H, Griffith J, Kurth T, Levey AS, Salem D, Sarnak MJ, Weiner DE. 2007. Cardiovascular disease and subsequent kidney disease. Arch. Intern. Med., 167(11): 1130-1136.

Friedewald WT, Levy RI, Fredrickson DS. 1972. Estimation of the concentration of low?density lipoprotein cholesterol in plasma, without use of the preparative ultracentrifuge. Clin. Chem., 18 (6): 499502.

Herget-Rosenthal S, Bokenkamp A, Hofmann W. 2007. How to estimate GFR-serum creatinine, serum cystatin $\mathrm{C}$ or equations? Clin. Biochem., 40: 153-161.

Klahr S, Levey AS, Beck GJ, Caggiula AW, Hunsicker L, Kusek JW, Striker G. 1994. The effects of dietary protein restriction and blood pressure control on the progression of chronic renal disease. $N$. Engl. J. Med., 330: 877-884.

Knight EL, Verhave JC, Spiegelman D, Hillege HL, de ZD, Curhan GC, de Jong PE. 2004. Factors influencing serum cystatin $\mathrm{C}$ levels other than renal function and the impact on renal function measurement. Kidney Int., 65: 1416-1421.

Larsson A, Malm J, Grubb A, Hansson LO. 2004. Calculation of glomerular filtration rate expressed in $\mathrm{mL} / \mathrm{min}$ from plasma cystatin C values in mg/L. Scand J. Clin. Lab. Invest., 64: 25-30. 
Levey AS, Coresh J, Balk E, Kausz AT, Levin A, Steffes MW, Hogg RJ, Perrone RD, Lau J, Eknoyan G. 2003. National kidney foundation practice guidelines for chronic kidney disease: evaluation, classification, and stratification. Ann Intern Med, 139: 137-147.

DOI:htt://dx.doi.org/10.7326/0003-4819139-2-200307150-00013

Levey AS, Stevens LA, Schmid CH, Zhang YL, Castro AF, Feldman HI, Kusek JW, Eggers P, Van Lente F, Greene T, Coresh J. 2009. A new equation to estimate glomerular filtration rate. Ann. Intern. Med., 150(9): 604-612.

Li HX, Xu GB, Wang XJ, Zhang XC, Yang JM. 2010. Diagnostic accuracy of various glomerular filtration rates estimating equations in patients with chronic kidney disease and diabetes. Chin. Med. J., 123: 745-751.

Ma YC, Zuo L, Chen JH, Luo Q, Yu XQ, Li Y, Xu JS, Huang SM, Wang LN, Huang W, Wang M, Xu GB, Wang HY. 2007. Improved GFR estimation by combined creatinine and cystatin $\mathrm{C}$ measurements. Kidney Int., 72: 1535-1542.

Macdonald J, Marcora S, Jibani M, Roberts G, Kumwenda M, Glover R, Barron J, Lemmey A. 2006. GFR estimation using cystatin $\mathrm{C}$ is not independent of body composition. Am. J. Kidney Dis., 48: 712719.

Matsushita K, Mahmoodi BK, Woodward M, Emberson JR, Jafar TH, Jee SH, Polkinghorne KR, Shankar A, Smith DH, Tonelli M, Warnock DG, Wen CP, Coresh J, Gansevoort RT, Hemmelgarn BR, Levey AS. 2012. Comparison of risk prediction using the CKD-EPI equation and the MDRD study equation for estimated glomerular filtration rate. JAMA, 307: 1941-1951.

Muntner P, Mann D, Winston J, Bansilal S, Farkouh ME. 2008. Serum cystatin C and increased coronary heart disease prevalence in US adults without chronic kidney disease. Am. J. Cardiol., 102: 5457.

Poggio ED, Wang X, Greene T, Van LF, Hall PM. 2005. Performance of the modification of diet in renal disease and Cockcroft-Gault equations in the estimation of GFR in health and in chronic kidney disease. J. Am. Soc. Nephrol., 16: 459-466.

Roos JF, Doust J, Tett SE, Kirkpatrick CM. 2007. Diagnostic accuracy of cystatin C compared to serum creatinine for the estimation of renal dysfunction in adults and children--a meta-analysis. Clin. Biochem., 40: 383-391.

Rosamond W, Flegal K, Friday G, Furie K, Go A, Greenlund K, Haase N, Ho M, Howard V, Kissela B, Kittner S, LloydJones D, McDermott M, Meigs J, Moy C, Nichol G, O'Donnell CJ. 2007. Heart disease and stroke statistics--2007 update: a report from the American Heart Association Statistics Committee and Stroke Statistics Subcommittee. Circulation, 115: e69-171.

Rule AD, Bergstralh EJ, Slezak JM, Bergert J, Larson TS. 2006. Glomerular filtration rate estimated by cystatin $\mathrm{C}$ among different clinical presentations. Kidney Int., 69: 399-405.

Séronie-Vivien S, Delanaye P, Pieroni L, Mariat C, Froissart M, Cristol J-P. 2008. Cystatine C : point d'étape et perspectives. Ann. Biol. Clin., 66(3): 301323.

Shimizu A, Horikoshi S, Rinnno H, Kobata M, Saito K, Tomino Y. 2003. Serum cystatin $\mathrm{C}$ may predict the early prognostic stages of patients with type 2 diabetic nephropathy. J. Clin. Lab. Anal., 17: 164-167.

Stevens LA, Coresh J, Schmid CH, Feldman HI, Froissart M, Kusek J, Rossert J, Lente FV, Bruce RD, Zhang Y, Greene T, Levey AS. 2008. Estimating GFR using serum cystatin $\mathrm{C}$ alone and in combination with serum creatinine: A 
pooled analysis of 3418 individuals with CKD. Am. J. Kidney Dis., 51(3): 395406.

Stevens LA, Li S, Kurella Tamura M, Chen SC, Vassalotti JA, Norris KC, WhaleyConnell AT, Bakris GL, McCullough PA. 2011. Comparison of the CKD Epidemiology Collaboration (CKDEPI) andModification of Diet in Renal Disease (MDRD) study equations. Am. J. Kidney Dis., 57(3) (suppl 2): S9-S16.

Stevens LA, Schmid CH, Greene T, Li L, Beck GJ, Joffe MM, Froissart M, Kusek JW,Zhang YL, Coresh J, Levey AS.
2009. Factors other than glomerular filtration rate affect serum cystatin C levels. Kidney Int., 75: 652-660.

Yang YS, Peng $\mathrm{CH}$, Lin $\mathrm{CK}$, Wang $\mathrm{CP}$, Huang CN. 2007. Use of serum cystatine $\mathrm{C}$ to detect early decline of glomerular filtration rate in type 2 diabetes. Intern. Med., 46: 801-806.

Zahran A, Qureshi M, Shoker A. 2007. Comparison between creatinine and cystatin C-based GFR equations in renal transplantation. Nephrol Dial Transplant, 22: $2659-2668$. 